\title{
Síntesis por implantación iónica de nanocristales semiconductores para dispositivos en tecnología de $\mathrm{Si}$
}

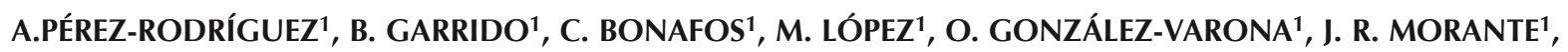 \\ J. MONTSERRAT ${ }^{2}$, R. RODRÍGUEZ ${ }^{3}$, J. A. GARCÍA-LORENTE ${ }^{3}$ \\ ${ }^{1}$ EME, Departament d'Electrònica, Unitat Associada CNM-CSIC, Universitat de Barcelona, Martí i Franqués 1, 08028 Barcelona \\ ${ }^{2}$ Centre Nacional de Microelectrònica, CNM-CSIC, Campus UAB, 08193 Bellaterra, \\ ${ }^{3}$ Asociación de la Industria Navarra (AIN), 31191 Cordovilla, Pamplona
}

\begin{abstract}
En este artículo se estudia la síntesis de nanocristales semiconductores elementales y compuestos elaborados por implantación iónica en $\mathrm{SiO}_{2}$. En el caso de los nanocristales de $\mathrm{Si}$, se ha desarrollado un estudio sistemático que correlaciona las características de los precipitados y sus propiedades de luminiscencia. Nanopartículas de Ge, que presentan menor emisión pero mayor contraste en Microscopía Electrónica de Transmisión, han sido fabricadas para desarrollar un nuevo método de medida de la densidad de nanocristales en matrices amorfas. Por otro lado, nanopartículas de ZnS dopadas con Mn han sido elaboradas por primera vez con esta técnica, observando la emisión de un pico de luminescencia característico de una transición intra-Mn. Finalmente, se presentan los primeros resultados ópticos de capas coimplantadas con $\mathrm{Si}^{+}$y $\mathrm{C}^{+}$, que muestran la presencia de tres picos intensos de luminescencia en las regiones roja, verde y azul del espectro visible, que ha sido relacionada con la presencia de diferentes tipos de nanopartículas. Cabe destacar que la emisión simultánea de los tres picos ha permitido la observación de una intensa emisión de luz blanca.
\end{abstract}

Palabras clave: nanopartículas semiconductoras, implantación iónica, luminescencia, Microscopía Electrónica de Transmisión

Ion Beam sysnthesis of semiconductor nanocrystals for Si technology devices

In this paper, we present the synthesis and the detailed characterisation of elemental and compound semiconducting nanoparticles ion beam synthesised in $\mathrm{SiO}_{2}$. In the case of $\mathrm{Si}$ nanoparticles, a systematic study has been developed in order to make the link between the characteristics of the precipitates and the optical properties. Ge nanoparticles, which emission is weak but having a strong contrast in Transmission Electron Microscopy, have been fabricated in order to develop a new method of precipitate density measurement. On the other hand, $\mathrm{ZnS}$ nanocrystals doped with $\mathrm{Mn}$ have been synthesised for the first time with this technique and show a PL emission centred at $2.12 \mathrm{eV}$, characteristics of an intra-Mn transition. Moreover, the first optical results of $\mathrm{Si}^{+}$and $\mathrm{C}^{+}$co-implanted layers are presented here and show three PL emissions, linked to the presence of three different types of nanoparticles. The simultaneous emission of these three peaks has allowed the observation of an intense white light.

Keywords: semiconducting nanoparticles, ion implantation, photoluminescence, Transmission Electron Microscopy

\section{INTRODUCCIÓN}

Desde el descubrimiento de la emisión radiativa del silicio poroso en 1990 (1), las nanopartículas semiconductoras elementales y compuestas han recibido un gran interés, como muestra el elevado número de publicaciones en la literatura dedicadas a la síntesis y estudio de estos sistemas. Esto ha venido motivado por su potencial para el desarrollo de dispositivos emisores de luz a temperatura ambiente y en el rango visible, compatibles con la tecnología de silicio. Estas estructuras presentan propiedades excepcionales de luminescencia en relación con el material macroscópico masivo, que resultan del confinamiento cuántico de los electrones y huecos en la nanopartícula. En el caso particular de semiconductores de "gap" indirecto, como el Si, la transición electrónica directa banda a banda puede ocurrir únicamente con la asistencia de fonones, lo que determina una baja probabilidad de recombinación radiativa. Pero para cristales de dimensiones comparables al radio de Bohr del excitón, el confinamiento espacial de los portadores de carga produce un desplazamiento en energía de la emisión de luz hacia el visible, así como un notable aumento de su eficiencia. Esto permite que nanocristales de silicio o de germanio, crecidos en una matriz de $\mathrm{SiO}_{2}$, emitan en el rango visible y a temperatura ambiente $(2,3)$.

Numerosas técnicas son utilizadas en la actualidad para elaborar estas estructuras. Entre ellas destaca la implantación iónica por su versatilidad y facilidad de control, lo que permite la síntesis de un amplio rango de nanocristales utilizando procesos compatibles con la tecnología de circuitos integrados de Si. Dentro de estos procesos, la utilización de una matriz de $\mathrm{SiO}_{2}$ es interesante, ya que éste es el material aislante por excelencia en la tecnología de $\mathrm{Si}$. Además, el $\mathrm{SiO}_{2}$ es transparente en el visible e infrarrojo cercano, y tiende a formar intercaras de buena calidad con los semiconductores. Se trata por tanto de un material muy bien conocido, compatible con la tecnología del silicio, fácil de elaborar a partir de substratos de Si, robusto y que presenta una pequeña concentración de defectos (4). 
En este contexto, el presente trabajo aborda la síntesis y caracterización detallada de nanocristales semiconductores elementales y compuestos elaborados por implantación iónica en $\mathrm{SiO}_{2}$. En el caso de los nanocristales de $\mathrm{Si}$, numerosos grupos de investigación han reportado la fabricación de estructuras luminescentes en el visible, pero el origen de la emisión de luz es todavía desconocido. Existe un amplio consenso en que, por efecto del confinamiento cuántico, el "gap" de las nanopartículas aumenta cuando disminuye su tamaño, pero la identificación del mecanismo de recombinación radiativa está sujeta a polémica. Entre los diferentes mecanismos propuestos, destacan: i) la recombinación radiativa banda -banda de los portadores $(5,6)$ y ii) la recombinación radiativa a través de estados localizados en la intercara nanocristal $/ \mathrm{SiO}_{2}(7,8)$. Independientemente de su origen, las propiedades ópticas y opto-electrónicas de estas estructuras nanocristalinas vienen directa o indirectamente determinadas por la estructura, densidad y tamaño de las nanopartículas. En este contexto, la originalidad de nuestro trabajo consiste en plantear un estudio sistemático que correlaciona las características de la población de precipitados en las capas procesadas y sus propiedades ópticas (9). Ello ha implicado la necesidad de establecer un nuevo método para la medida de la densidad de nanopartículas en matrices amorfas. Este método, basado en técnicas de Microscopía Electrónica, ha sido desarrollado utilizando nanopartículas de $\mathrm{Ge}$ en $\mathrm{SiO}_{2}$, que, constituyen un sistema especialmente adecuado para su observación en el microscopio electrónico $(10,11)$, aunque presentan en general una eficiencia de luminescencia baja en el visible.

Por otra parte, la flexibilidad de la implantación iónica permite plantear la síntesis de nanocristales de semiconductores compuestos. La inclusión de estas nanopartículas en capas de $\mathrm{SiO}_{2}$ posibilitaría la integración de materiales interesantes en aplicaciones optoelectrónicas en tecnología de Si. En este marco, el primer objetivo planteado ha sido la síntesis de nanocristales de $\mathrm{ZnS}$ y $\mathrm{SiC}$ mediante procesos de implantación secuencial. En el primer caso, hemos reportado la formación por implantación iónica, por primera vez en la literatura, de nanocristales de ZnS dopados con Mn $(12,13)$. Los espectros de luminescencia de estas capas presentan un pico de emisión a $2.12 \mathrm{eV}$, que es característico de una transición intra-Mn, lo que demuestra su activación como centro de color en los nanocristales. En el segundo caso se presenta la coimplantación de $\mathrm{Si}^{+}$y $\mathrm{C}^{+}$en $\mathrm{SiO}_{2}$. Los resultados previos obtenidos muestran la existencia de un espectro de emisión más complejo, con picos intensos en las zonas roja, verde y azul del espectro visible que están relacionados con la formación de diferentes tipos de nanopartículas.

\section{PROCEDIMIENTO EXPERIMENTAL}

Los substratos utilizados en este trabajo son obleas de Si (100), que han sido oxidadas térmicamente para formar capas de $\mathrm{SiO}_{2}$ entre 400 y $800 \mathrm{~nm}$ de grosor. Los procesos de implantación han sido realizados a temperatura ambiente, con dosis en un amplio rango entre $10^{16}$ y $3 \times 10^{17}$ iones $/ \mathrm{cm}^{2}$ y energías entre 60 y $150 \mathrm{keV}$, en función de la especie implantada. Para formar los compuestos, las energías de implantación han sido escogidas para obtener el solapamiento máximo de los perfiles y la secuencia de implantación ha sido determinada a partir de un estudio termodinámico previo. Los parámetros de cada implantación han sido definidos a partir del simulador TRIM (14). Las muestras han sido recocidas en una amplia gama de

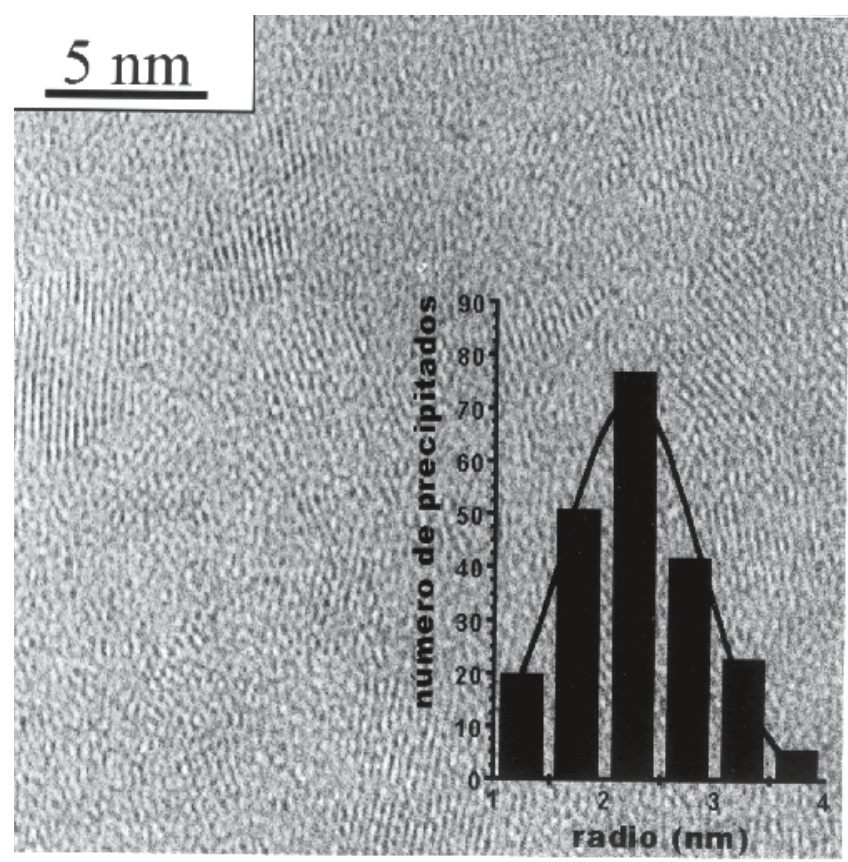

Fig. 1: Imagen de Microscopía Electrónica de Transmisión de los nanocristales de $\mathrm{Si}$ en $\mathrm{SiO}_{2}$ y distribución de tamaño asociada.

temperaturas entre 500 y $1200^{\circ} \mathrm{C}$ en atmósfera de $\mathrm{N}_{2}$, en hornos de tipo "Rapid Thermal Annealing" (RTA) para tiempos de recocidos inferior a 10 minutos y en hornos convencionales para tiempos de recocidos más largos. El estudio de las cinéticas de crecimiento de los nanocristales ha sido realizado a partir de la observación por Microscopía Electrónica de Transmisión (TEM) en sección transversal y Alta Resolución, utilizando un microscopio Philips CM-30 a 300 keV. La caracterización estructural ha involucrado, cuando ha sido necesario, un amplio abanico de técnicas como la espectroscopia de pérdida de energía de los electrones (EELS), Espectroscopía Raman e Infrarrojo, Espectroscopía de fotoelectrones de rayos X (XPS), Resonancia Paramagnética Electrónica (EPR) y Espectroscopía de Masa de Iones Secundarios (SIMS). Finalmente, los espectros de Fotoluminescencia (PL) han sido medidos a temperatura ambiente excitando las muestras con un láser UV de He-Cd $(\lambda=325 \mathrm{~nm})$.

\section{NANOCRISTALES ELEMENTALES DE “GAP" INDIRECTO (SI, GE)}

\subsection{Nanocristales de $\mathrm{Si}$ en $\mathrm{SiO}_{2}$}

$\mathrm{El}$ espectro de luminescencia de las capas de $\mathrm{SiO}_{2}$ implantadas con Si depende de gran medida de la temperatura de recocido: para temperaturas inferiores a $950^{\circ} \mathrm{C}$, la separación de fase se produce y la capa implantada es una solución sólida sobresaturada de $\mathrm{Si}$ en $\mathrm{SiO}_{2}$. Se observan en este caso tres picos de PL en el visible, que han sido identificados con la presencia de tres tipos de defectos en el $\mathrm{SiO}_{2}$, inducidos por la implantación (9). La intensidad de estos picos disminuye al aumentar la temperatura de recocido, desapareciendo para temperaturas superiores a $950^{\circ} \mathrm{C}$. En este caso, tiene lugar la separación de fases con la formación de nanocristales de Si, como se puede ver en la Fig. 1. Esta nucleación viene acompañada de la apa- 
rición de un pico de PL, muy intenso, centrado en $1.6 \mathrm{eV}$ y con una anchura a media altura de $0.3 \mathrm{eV}$, tal como se muestra en el espectro insertado en la Figura 2. Las características de este pico han sido estudiadas en función de las condiciones de recocido (tiempo de recocido, con una temperatura fija de $1100^{\circ} \mathrm{C}$ ) en muestras implantadas con una concentración de Si máxima inicial del $10 \%$ atómica (9). Este estudio muestra que la energía de la emisión no depende del tiempo de recocido, que por otra parte determina el tamaño de los nanocristales. Esto sugiere la existencia de un mecanismo de recombinación radiativa a través de estados localizados en la intercaras nanocristal $/ \mathrm{SiO}_{2}(7,8)$. Por otra parte, la intensidad del pico muestra un fuerte aumento al aumentar el tiempo de recocido hasta tiempos de 2 horas, produciéndose después una saturación, tal como se muestra en la Fig. 3. El análisis de las muestras por EPR muestra que la intensidad de pico de PL varía de forma inversa con la concentración de centros $\mathrm{Pb}$, que corresponden a enlaces libres de $\mathrm{Si}$ en la superficie de los nanocristales (ver Fig. 3). Este comportamiento constituye una evidencia experimental del papel de los defectos $\mathrm{Pb}$ como centros de recombinación no radiativa, que compiten con la recombinación radiativa responsable del pico de PL observado. Estos resultados son compatibles con el modelo desarrollado por Lannoo et al (4) de atrapamiento del excitón en enlaces Si-Si tensionados en la región interfacial, como consecuencia de la diferencia de longitud entre los enlace Si-Si y Si-O. Es importante destacar que este comportamiento depende también del nivel inicial de sobresaturación de las capas implantadas con $\mathrm{Si}$, siendo el comportamiento observado característico de niveles altos de sobresaturación. La dependencia del mecanismo dominante de recombinación del nivel inicial de sobresaturación de Si permite entender la existencia de resultados contradictorios en la literatura.

\subsection{Nanocristales de $\mathrm{Ge}$ en $\mathrm{SiO}_{2}$}

Tal como se ha indicado previamente, estructuras con nanocristales de Ge también han sido sintetizadas por implantación iónica. En este caso, y con el fin de minimizar posibles efectos de difusión, se ha realizado una implantación múltiple (en cuatro etapas) para obtener una capa implantada con una concentración uniforme de Ge, del orden del $5 \%$ atómico. Después de un recocido a temperaturas superiores a $900^{\circ} \mathrm{C}$, se observa la separación de fase con la consiguiente formación de nanocristales de Ge. Estos nanocristales emiten también en el rango visible $(2.3 \mathrm{eV})$, pero con una intensidad muy baja, en el límite de resolución del detector. No obstante, este sistema nos ha permitido desarrollar un estudio completo de la cinética de crecimiento de los nanocristales en la matriz amorfa en función de los parámetros de recocido.

Para ello, se ha realizado una caracterización detallada de la distribución en tamaño de los nanocristales a partir de las imágenes de TEM de sección transversal. Los histogramas de tamaño han sido extraídos de las imágenes en campo claro desfocalizadas (Fig. 4), que dan resultados más fiables que las imágenes en contraste de fase de alta resolución $(10,11)$. Por otra parte, la naturaleza amorfa de la matriz de $\mathrm{SiO}_{2}$ impide la determinación directa del espesor de la región observada, que es necesario para determinar la densidad de precipitados (cantidad de precipitados por unidad de volumen). Por ello, se ha desarrollado un nuevo método, utilizable de manera sistemática, para medir la densidad volumétrica de precipitados, que combina TEM convencional con espectroscopía EELS. En esta

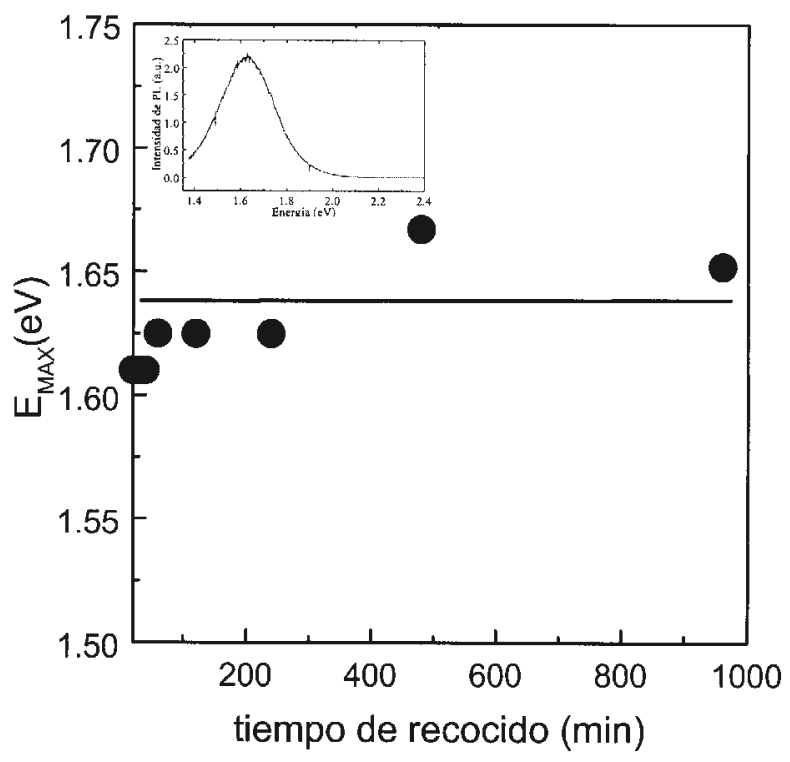

Fig. 2: Evolución con el tiempo de recocido de la energía de PL para la muestra implantada con $10 \%$ atómico de Si y recocida a $1100^{\circ} \mathrm{C}$.

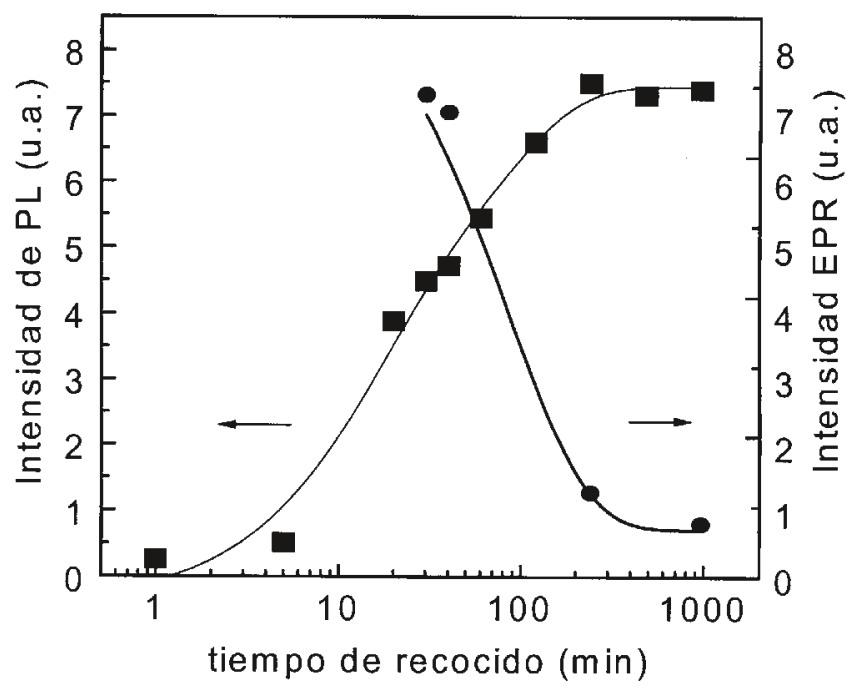

Fig. 3: Evolución con el tiempo de recocido de la intensidad de la PL y de la concentración de centros $\mathrm{Pb}$ medida con EPR.

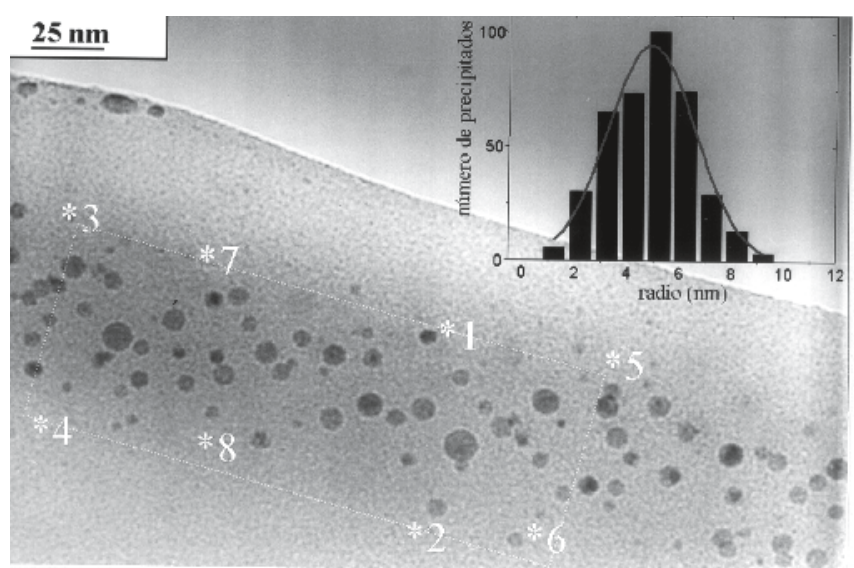

Fig. 4. Imagen de Microscopía Electrónica de Transmisión en condiciones de campo claro desfocalizado de los nanocristales de Ge en $\mathrm{SiO}_{2}$ indexada en grosor por EELS (ei=14,64,19,70,27,63,14,68 nm) y distribución de tamaños asociada. 


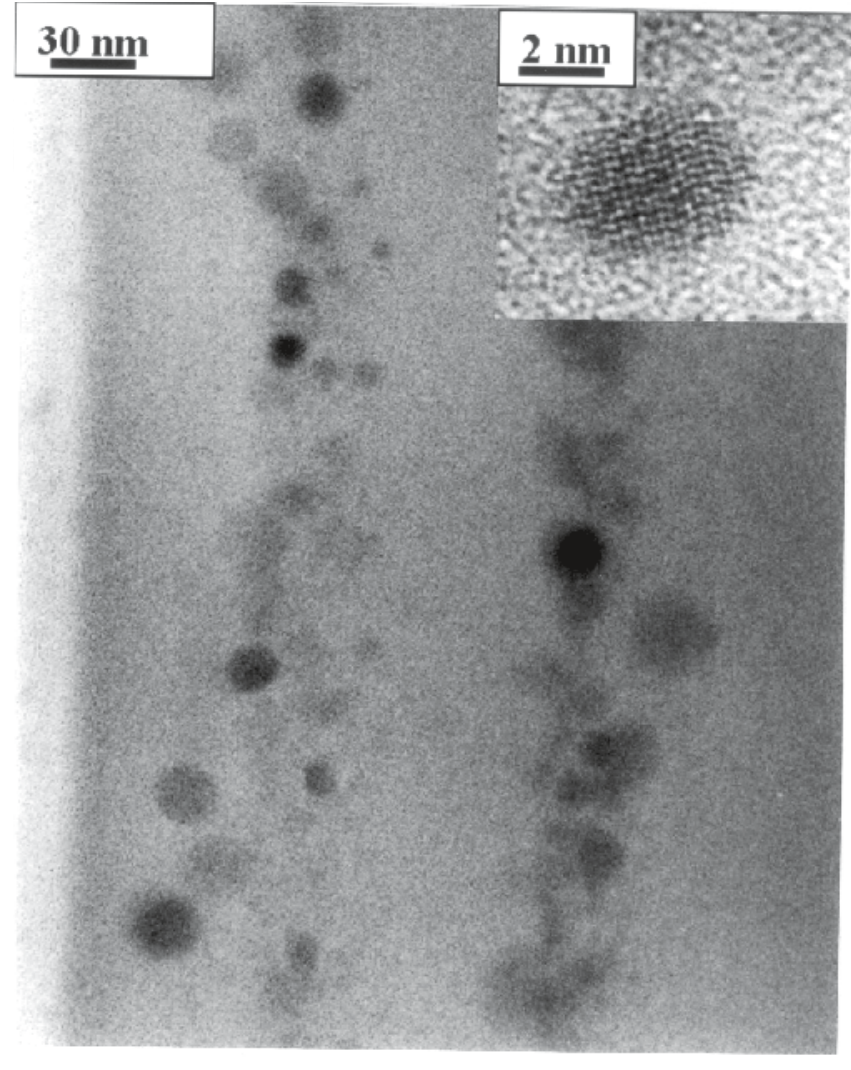

Fig. 5. Imagen de Microscopía Electrónica de Transmisión con una distribución bimodal de los nanocristales de ZnS. En el cuadro superior se muestra la imágen en alta resolución de un nanocristal.

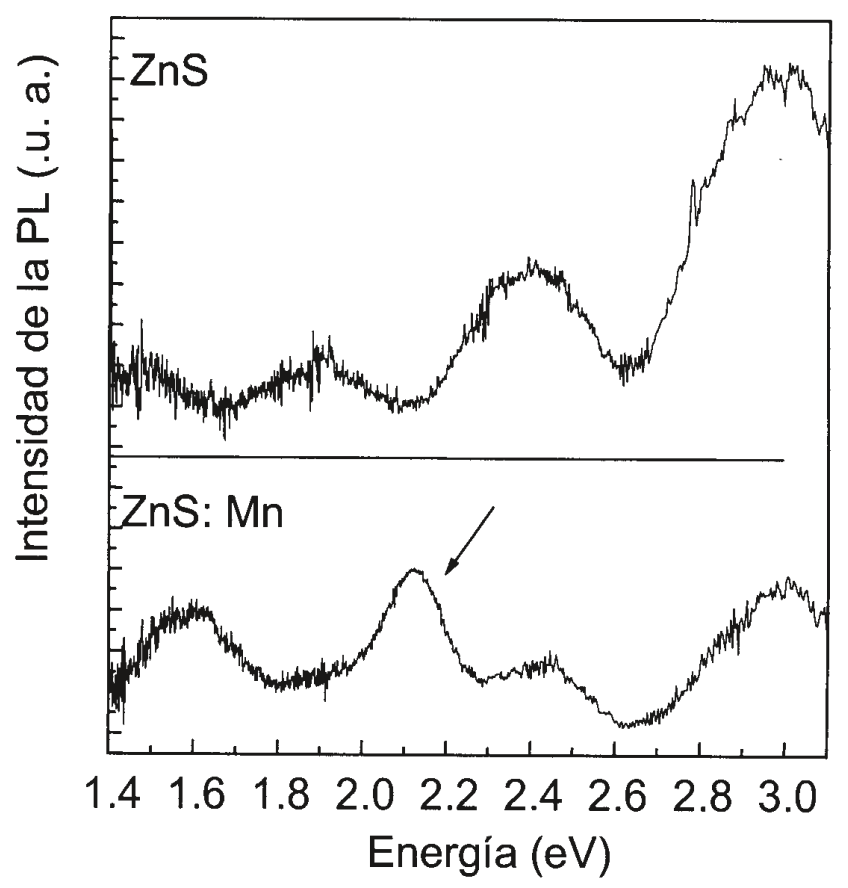

Fig. 6. PL emitida por (a) los nanocristales de ZnS y (b) los nanocristales de $\mathrm{ZnS}$ dopados con Mn. técnica, el espesor en diferentes lugares de la imagen ha sido estimado mediante el ajuste y relación de los picos sin pérdida y con pérdidas de energía en el espectro EELS correspondiente. De esta manera, se puede realizar una topografía de las imágenes donde se pueden contar los precipitados, tal como se muestra en la Fig. 4. Estas medidas han permitido obtener la evolución de la distribución de tamaño, la densidad de precipitados y el número de átomos de Ge dentro de los precipitados con el tiempo de recocido a una temperatura fija de $1000^{\circ} \mathrm{C}$. La comparación de las leyes de crecimiento experimentales con las previstas por la teoría de la precipitación ha mostrado que los nanocristales están en fase de maduración Ostwald (11). La etapa siguiente consistirá en aplicar este método de medida de la densidad de precipitados al sistema $\mathrm{Si} / \mathrm{SiO}_{2}$, que resulta mucho más interesante desde el punto de vista de la emisión luminescente, y en el que las medidas son más problemáticas debido al bajo nivel de contraste entre las fases $\mathrm{Si} \mathrm{y} \mathrm{SiO}_{2}$.

\section{NANOCRISTALES COMPUESTOS}

\subsection{Nanocristales de "gap" directo: precipitados de ZnS dopados con Mn}

La síntesis de semiconductores compuestos presenta un nivel mayor de complejidad, y requiere del estudio previo de las posibles interacciones a nivel químico entre las especies implantadas y los átomos de la matriz. La viabilidad desde el punto de vista termodinámico de la síntesis de la especie propuesta puede ser estudiada mediante la aplicación del criterio de Hosono (15). Esto ha permitido comprobar que la formación por implantación iónica y tratamiento térmico de nanocristales de $\mathrm{ZnS}$ es energéticamente posible, teniendo lugar mediante la reacción $2 \mathrm{ZnO}+\mathrm{SiS}_{2} \longrightarrow 2 \mathrm{ZnS}+\mathrm{SiO}_{2}$ (12). El ZnS es un semiconductor de "gap" ancho directo que emite en la región UV del espectro, que es utilizado para la fabricación de dispositivos emisores de luz, debido a la posibilidad de introducir centros de color que emiten en el visible (16) como el Mn para la emisión en el verde-amarillo.

La caracterización estructural de las capas recocidas ha corroborado la formación de nanocristales de ZnS para temperaturas de recocido superiores a $900^{\circ} \mathrm{C}$. Los resultados de difracción de electrones y de Rayos X coinciden al concluir que estos nanocristales tienen una estructura tipo wurtzita-2H $(12,13)$. La observación por TEM de la sección transversal de las capas implantadas muestra la organización de los precipitados en dos capas paralelas a la superficie de la muestra (Fig. 5). Esto coincide con la aparición en los espectros SIMS correspondientes de dos picos en los perfiles de $S$ y de $\mathrm{Zn}$, centrados en las capas de precipitados. Esta organización de los precipitados puede tener dos orígenes distintos: i) la existencia de un proceso de crecimiento competitivo $(12,13)$ y ii) una nucleación heterogénea en las regiones de máximo daño por el proceso de implantación.

El espectro de luminescencia de estas capas muestra la presencia de cuatro picos de baja intensidad, centrados en $1.5 \mathrm{eV}$, $1.9 \mathrm{eV}, 2.4 \mathrm{eV}$ y $2.9 \mathrm{eV}$ (Fig. 6 (a)). El origen de estos picos no está claro, y podría estar relacionado con la presencia de defectos en el óxido (13). La implantación de estas capas con iones de Mn previamente a la etapa de recocido induce la aparición de un pico adicional, centrado en $2.12 \mathrm{eV}$ (Fig, 6 (b)). Esto coincide con el valor teórico de la transición intra-Mn ${ }^{4} \mathrm{~T}_{1}-{ }^{6} \mathrm{~A}_{1}$, lo que demuestra el dopado de los nanocristales de $\mathrm{ZnS}$ con el Mn implantado. 


\subsection{Nanocristales de "gap" indirecto: precipitados de SiC}

Finalmente, se ha abordado la síntesis por implantación iónica de nanopartículas de $\mathrm{SiC}$. El trabajo previo en estos sistemas ha permitido observar la existencia de procesos de exodifusión del carbono durante los tratamientos de recocido en horno convencional, que han sido interpretados como consecuencia de la formación y posterior exodifusión de CO $(4,17)$. Para evitar este efecto, se ha realizado una implantación múltiple de $\mathrm{Si}$ (en dos etapas) que da lugar a una capa enterrada con un contenido uniforme de $\mathrm{Si}$ en la matriz de $\mathrm{SiO}_{2}$. Posteriormente, se ha realizado una implantación de iones $\mathrm{C}$, seleccionando la energía de implantación para obtener la distribución de carbono dentro de la capa rica en Si. Esto permite minimizar la formación de enlaces C-O en la capa implantada, y la consiguiente migración del carbono hacia la superficie. En una primera fase, se han seleccionado los parámetros de la implantación para obtener una concentración de $\mathrm{Si}$ en la capa enterrada del $20 \%$ atómico, que coincide con la concentración máxima del perfil de C implantado.

El estudio de la luminescencia de estas capas muestra la presencia de tres picos intensos en el espectro visible, cuya resultante es una luz blanca muy fuerte. Estos tres picos están centrados respectivamente a $1.5 \mathrm{eV}$ (rojo), $2.25 \mathrm{eV}$ (verde) y $3.1 \mathrm{eV}$ (azul) como se puede ver en la Fig. 7. El estudio preliminar de los procesos de co-implantación de $\mathrm{Si}^{+}$y $\mathrm{C}^{+}$en $\mathrm{SiO}_{2}(4,17)$ ha permitido identificar el origen de estos picos, que está relacionada con la formación de diferentes tipos de nanopartículas: el pico rojo ha sido atribuido a la presencia de nanopartículas de Si. Por otra parte, el pico verde ha sido correlacionado con la formación de precipitados grafíticos de carbono, cuya presencia ha sido detectada mediante espectroscopía Raman. Por último, el pico azul ha sido atribuido a la formación de precipitados de $\mathrm{SiC}$, a partir de la observación de una contribución de Si coordinado tetraédricamente a $\mathrm{C}\left(\mathrm{Si}-\mathrm{C}_{4}\right)$ y C coordinado tetraédricamente a $\mathrm{Si}\left(\mathrm{C}-\mathrm{Si}_{4}\right)$ en los espectros $\mathrm{Si} 2 \mathrm{p}$ y $\mathrm{C} 1 \mathrm{~s}$ de XPS.

\section{CONCLUSIONES}

Este trabajo ha demostrado la potencialidad de los procesos de implantación iónica para la síntesis de una gran variedad de nanopartículas con propiedades de luminiscencia en substratos compatibles con tecnología de Si. Esto ha incluido tanto semiconductores elementales ( $\mathrm{Si}, \mathrm{Ge}$ ) como compuestos de "gap" directo (ZnS) o indirecto ( $\mathrm{SiC}$ ), y los procesos han incluido también el dopado efectivo de los nanocristales con centros de color (Mn:ZnS). Desde del punto de vista de la luminiscencia, los sistemas con mayor eficiencia son el $\mathrm{Si}_{\mathrm{SiO}}$ y $(\mathrm{Si}+\mathrm{C}): \mathrm{SiO}_{2}$, siendo en estos casos la emisión observable a simple vista. El análisis estructural detallado de las capas, que se está desarrollando en función de su procesado, permitirá clarificar la asignación de los picos de emisión fundamentales observados con la formación de nanopartículas de diferente composición ( $\mathrm{Si}, \mathrm{C}$ y $\mathrm{SiC}$ ), así como identificar los mecanismos de recombinación radiativa responsables de la emisión en cada caso.

\section{AGRADECIMIENTOS}

Este trabajo ha sido financiado por la CICYT, dentro del proyecto MAT97-0377-C02-01. Uno de los autores (C.B.) agradece la financiación recibida de la Unión Europea para su estancia en el Laboratorio EME, dentro del programa "Training and Mobility for Researchers" (proyecto ERBFMBICT 97 2279).

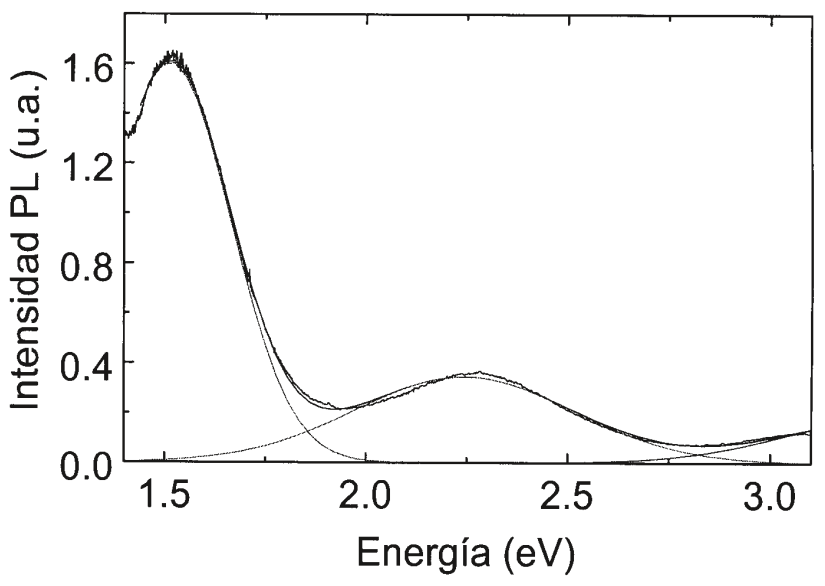

Fig. 7. Emisión de PL de las muestras co-implantadas con $20 \%$ atómico de Si y C.

\section{BIBLIOGRAFÍA}

1. L. T. Canham, "Silicon quantum wire array fabrication by electrochemical and chemical dissolution of wafers", Appl. Phys. Lett. 57, 1046-1048 (1990).

2. T. Shimizu-Iwayama, M. Ohshima, T. Niimi, S. Nakao, K. Saitoh, T. Fujita y N. Itoh, "Visible photoluminescence related to $\mathrm{Si}$ precipitates in $\mathrm{Si}^{+}$implanted $\mathrm{SiO}_{2}$ " J. Phys. Cond. Mat. 5, L375-380 (1993).

3. Y. Maeda, N. Tsukamato, Y. Yasawa, Y. Kanemitsu y Y. Masumoto," "Visible photoluminescence of Ge microcrystals embedded in $\mathrm{SiO}_{2}$ glassy matrices", Appl. Phys. Lett. 59, 3168 (1991).

4. B. Garrido, C. Bonafos, M. López, A. Cuadras, A. Pérez-Rodríguez y J. R. Morante, "On the incorporation of carbon in $\mathrm{SiO}_{2}$ layers", Defects and Diffusion Forum 160-161, 1-24 (1998).

5. P. F. Trowga, A. J. Kenyon y C. W. Pitt, "Modeling the contribution of quantum confinement to luminescence from silicon nanoclusters", J. Appl. Phys. 83, 37893794 (1998).

6. K. B. Kurgin, E. W. Forsythe, G. S. Tompa y B. A. Khan, "Influence of the size dispersion on the emission spectra of the Si nanostructures", Appl. Phys. Lett. 69, 1241-1243 (1996).

7. F. Koch, V. Petrova-Koch y T. Muschik, "The luminescence of porous Si: the case of the surface state mechanism", Journal of Luminescence 57, 271-281 (1993).

8. C. Delerue, G. Allan y M. Lanoo, "Theoretical aspects of the luminescence of porous silicon", Phys. Rev. B 48, 11024-11035 (1993).

9. M. Lopez, B. Garrido, C. Bonafos, A. Perez-Rodriguez, J. Montserrat y J. R. Morante, "The role of the surface of Si nanocrystals embedded in $\mathrm{SiO}_{2}$ on the visible emission", European Materials Research Society 1999, Spring Meeting, Estrasburgo (Francia), Junio 1999.

10. C. Bonafos, M. Lopez, B. Garrido, A. Perez-Rodriguez, J. R. Morante, J. Montserrat, M. Toufella, Y. Kihn, G. Ben Assayag, A. Claverie, A. Nejim y P. L. F. Hemment, "A kinetical study of the growth of Ge precipitates in $\mathrm{SiO}_{2}$ by coupling TEM and EELS", Proceedings of the $11^{\text {th }}$ International Conference on Microscopy of Semiconducting Materials, Institute of Physics Conf. Ser. 1999 (en prensa)

11. C. Bonafos, B. Garrido, M. Lopez, A. Perez-Rodriguez, J. R. Morante, Y. Kihn, G. Ben Assayag y A. Claverie, "Ostwald ripening of Ge precipitates elaborated by ion implantation in $\mathrm{SiO}_{2}{ }^{\prime \prime}$, Materials Science \& Engineering B 69-70, 379-384 (1999).

12. C. Bonafos, B. Garrido, M. Lopez, A. Romano-Rodriguez, O. Gonzalez-Varona, A. Perez-Rodriguez y J. R. Morante, "Ion Beam Synthesis and structural characterization of ZnS nanocrystals", Appl. Phys. Lett. 72-75, 3488-3490 (1998).

13. C. Bonafos, B. Garrido, M. Lopez, A. Romano-Rodriguez, O. Gonzalez-Varona, A. Perez-Rodriguez, J. R. Morante y R. Rodriguez "Structural and optical characterization of $\mathrm{Mn}$ doped $\mathrm{ZnS}$ nanocrystals elaborated by ion implantation in $\mathrm{SiO}_{2}{ }^{\prime \prime}$, Nucl. Instr. Meth. Phys. Res. B 147, 373-377 (1999).

14. F. Ziegler, J.P. Biersack, y U. Littmark, "The stopping of ions in solids", Pergamon Press, New York, 1985.

15. H. Hosono, "Simple criterion on colloid formation in $\mathrm{SiO}_{2}$ glasses by ion implantation", Jpn. J. of Appl. Phys. 32, 3892-3894 (1993).

16. R. Mach y G. O. Müller, "Physical concepts of High-Field thin-film Electroluminescence devices", Phys. Stat. Sol. 69, 11-30 (1982).

17. A. Perez-Rodriguez, B. Garrido, C. Bonafos, M. Lopez, O. Gonzalez-Varona, J Montserrat, R. Rodriguez y J. R. Morante, “Ion Beam synthesis of compound nanoparticles in $\mathrm{SiO}_{2}{ }^{\prime \prime}$, J. Mater. Scien.: Materials in Electronics 10, 385 (1999). 